Pak. j. sci. ind. res. Ser. B: biol. sci. 2020 63B(2) 127-131

\title{
Review
}

\section{A Short Review of Promoters of Cotton Fibre Genes: Strength and Tissue Specificity}

\author{
Ammara Masood ${ }^{a *}$ Nadia Iqbal $^{\mathrm{b}}$ and Hira Mubeen ${ }^{\mathrm{a}}$ \\ ${ }^{a}$ University of Central Punjab, Lahore, Pakistan \\ ${ }^{b}$ Muhammad Nawaz Shareef University of Agriculture, Multan, Pakistan
}

(received December 20, 2017; revised June 6, 2018; accepted July 2, 2018)

\begin{abstract}
Promoters are of great importance for controlling gene expression. There are various regulatory regions within promoters which are crucial for expression specificity. Depending upon mode of expression, promoters can be divided into constitutive, tissue/developmental stage specific or inducible promoters. For expression of certain gene, there is interplay of cis acting element located on promoter and transcription factors. Cotton fibre promoters are of great importance in biotechnology as these may be used to express fibre genes in cotton fibres specifically. Although most of cotton fibre gene promoters show expression in fibres but some of them also exhibit expression in various other organs like trichomes, pedicles and vascular tissues. Due to difficulties in somatic embryogenesis in cotton, these promoters are mostly studied in heterologous systems like Arabidopsis and tobacco. In this article, expression pattern of some cotton fibre genes promoters have been reviewed.
\end{abstract}

Keywords: cotton fibre genes, promoters, Arabidopsis, tobacco, dicots, tissue specific expression

\section{Introduction}

Promoters. Promoter is upstream region of gene through which RNA polymerase-II binds and initiates transcription. Various transcription factors interact with these regulatory sequences and alter the expression of gene (Gou, 2007). A promoter comprises of various regulatory regions which influence the rate of transcription including transcription initiation site and cis acting elements scattered on whole promoter region (McKnight and Tjian, 1986). Depending upon distance from transcription start site promoter can be divided into two regions; core promoter and distal promoter. The core promoter region is site for binding of RNA polymerase-II with general transcription factors to form transcription pre initiation complex (ASO et al., 1994). A crucial core promoter motif TATA box (TATAAAAAA) is located in the core region where TATA binding proteins (TBP) bind along with protein TF including TFIID, TFIIB. CAAT box is also located in core promoter region which is essential for promoter strength.

Distal promoter region may be thousands of base pairs away from transcription initiation site.

Core promoter contains TATA box while, distal promoter

*Author for correspondence;

E-mail: ammara.masood@ucp.edu.pk contains cis acting regulatory elements. Distal region is thousands of base pairs away from transcription start machinery.

Types of promoter. Promoters can be divided depending upon their mode of controlling gene expression including constitutive, tissue and developmental stage specific and inducible promoters.

Constitutive promoters are used to derive expression of gene in all tissues and during all developmental stages across species and genera. Most commonly used are promoters for dicot expression to derive constitutive expression include $35 \mathrm{~S}$ or $19 \mathrm{~S}$ promoters from Cauliflower Mosaic virus (Benfyet al., 1990). Promoters other than viral origin are octopine synthase promoter (Ellis et al., 1987) and nopaline synthase promoter (An et al., 1986) used to express in heterologous system.

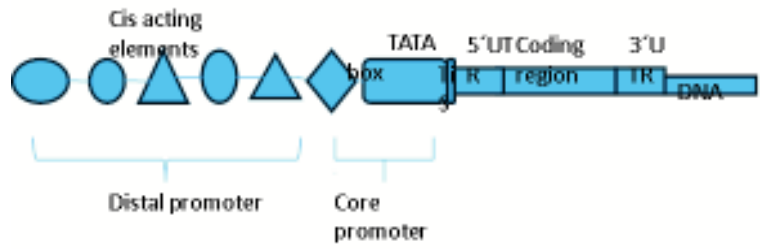

Fig 1. Promoter regions in relation to distance from transcription initiation site. 
These promoters have enhancer regions for binding of transcription factors and of transcription. On the other hand, tissue specific promoters have certain motifs essential for tissue specificity. These include PEP carboxykase active in photosynthetic organs, E8 a fruit specific promoter (He et al., 2008) and $L E A F Y$ promoter (Douliez, 2000). Inducible promoters and tissue specific promoters modulate gene expression not during all conditions but under specific biotic or abiotic stresses or certain tissues. Some examples of inducible promoters include promoter of maize sucrose synthase 1 gene (Yang and Russell, 1990) and sweet potato peroxide promoter (Kim et al., 2003).

Along with these categories hybrid and synthetic promoter are also being used to derive gene expression. These promoters are synthesized by assembling various regulatory regions of promoter without disturbing their functionality (Salinas et al., 1992). Hybrid promotes are synthesized by fusion of promoter sequences and functional domains from two or more distinct sources (Masood et al., 2017). The objective to generate hybrid promoter is over rule patency issues.

Cis-acting regulatory elements. Whether constitutive or tissue specific, all promoters have specific motifs scattered in whole promoter region. These include $W$ box (TTGACC/T), dehydration response elements (Ruston et al., 2010), ABA responsive elements (Deikman et al., 1998) and ethylene responsive elements (Gou et al., 2008). These motifs are related to stress responsive expression of promoter. There are various light responsive motifs including $G$ box, $F$ box (Paquis, 2010) and $I$ box (Guiliano et al., 1988). G box present in the prompters of light regulated genes also exhibit organ specific expression (Salinas et al., 1992). There are few available data bases those provide information about promoter $c i s$-acting elements and transcription factor binding sites for promoters. These include Plant CARE (Lescot et al., 2002), TRANSFAC (Wingender, 2001) transcription regulatory region data base (TRRD) (Kolchanov et al., 2002).

For transformation of useful genes in plants, a variety of promoters are desirable to prevent any genetic recombination caused by identical sequences. In cotton, promoter study helps to understand the mechanism of gene regulation in fibre cells. Therefore, there is need for novel promoters which are organ, developmental stage specific or activated by external stimuli. A large number of constitutive promoters have been isolated and being used in molecular research. But use of constitutive promoter may cause unnecessary metabolic burden on plant or their product may be accumulated that may cause deleterious effect on plant life. In contract, tissue specific promoter express specific genes with controlled expression for recombinant product.

Cotton fibre promoters. A number of fibre specific promoters have been discovered (Qin et al., 2013) in cotton. Transgenic plants transformed with majority of these promoters have showed tissue specific expression especially in trichomes. These fibre specific promoters are active during certain period of fibre growth and may be used to alter gene expression during certain fibre developmental phase. A fiber specific promoter isolated from up stream region of $E 6$ gene was found to express specifically in fibre (John and Crow, 1992). John promoter of E6 gene was used to drive fibre specific expression for modification of cotton fibre quality (John and Keller, 1996). An up-stream region of another $E 6$ gene was evaluated as promoters which exhibited trichome specific expression using GUS and GFP reporter genes (Aimin and Jinyuan, 2005). Upstream regions of various $L T P$ genes have also been showed tissue specific expression (Wu et al., 2007). Promoter of cotton beta galactosidase gene (GhGal1) exhibited expression in various tissues of transgenic tobacco including roots, vascular tissues, trichomes and fruits). Sequence analysis revealed many already reported motifs in promoter sequence (Wu et al., 2006).

Li et al. (2002) isolated a promoter of fibre specific TUB1 gene which showed fibre specific expression. Promoter of cotton reversibly glycosylated polypeptides exhibited tissue specific as a well as induced expression (Wu et al., 2006). Up-stream region of an arabinoglactan GhH6L gene showed GUS expression in the pedicle and petiole of tobacco (Wu et al., 2009). Promoter of $M Y B$ transcription factor genes including $M Y B 2$, MYB109 and GhMYB25 involved in fibre development also exhibited GUS expression in tobacco trichomes as well as fibre tissues (Pu et al., 2008). A cotton protodermal factor $G b P D$ gene promoter showed GUS expression on ovular surface and developing fibres. Deletion analysis revealed a HDZIP2ATATHB2 element found to essential for promoter activity (Deng et al., 2012).

A novel cotton fibre specific promoter GhSCFP was isolated from a fibre specific gene was found to derive fibre specific expression from initiation till maturation 
stage (Hou et al., 2008). A promoter region of fibre specific gene $F b L 2 A$ isolated from sea land cotton Gossypium barbadense was found to be expressed during primary and secondary cell wall synthesis stage of fibre development. This promoter was found to derive fibre specific expression of genes coding for acetoacetylco-enzyme reductase and poly hydroxyalkanoic acid synthase. A cotton fibre glucronosyl-transferase gene ( Gh GlcAT1) promoter was found to express in root cap, seed coat and trichomes of transgenic tobacco. Deletion analysis of promoter revealed many regulatory motifs involved in the trichome specific expression, sugar and stress responsiveness (Wu et al., 2007). Fibre specific promoters may regulate the process of fibre development by expressing genes in fibre cells during distinct phase of fibre development. These promoters can be used for expression of fibre specific genes for development of transgenic cotton (Li et al., 2005). To analyze expression of cotton fibre specific promoters in relatively shorter time a novel expression system of cultured cotton ovules has been optimized (Iqbal et al., 2017).

Along with fibre specific expression, various promoters of fibre related gene can derive vascular tissues specific expression. These promoters can be used to express defense genes for resistance against pathogen attack which are translocated through vascular tissues of plants. These promoters can be used to block the viral or other pathogen movement in vascular tissues. These tissue specific promoters may use coupled with transgene for specific expression in heterologous system (Renihart et al., 1996). For example, tomato pollen specific promoter expresses in tobacco (Twell, 1990). Promoters of lipid transfer proteins show trichome specific expression in tobacco (Liu et al., 2000), Arabidopsis pGc1 promoter shows specific expression in Arabidopsis as well as tobacco guard cells (Yang et al., 2008 ).

\section{Conclusion}

Promoters are not only important for gene expression but also help to understand gene function. A large variety of promoters are used to express genes in plants. Along with constitutive and inducible promoter, tissue specific promoters are of great importance the express genes in controlled manner. In cotton, fibre specific promoter may help to express gene in fibre tissues to improve fibre quality. A number of promoters of fibre genes have been isolated as up-stream regulatory sequences from cotton genome. Bio-informatics tools help to identify promoters and their regulatory elements. The up-stream region of various genes have been identified and analy-zed for promoter activity. Through expression analysis of genes, their upstream sequences may also be selected and further evaluated for promoter expression and strength. Promoters of cotton fibre genes including E6, lipid transfer proteins, expansions, XTH and MYB proteins show expression in fibre and other cotton tissues. These promoters also show expression in various organs of Arabidopsis and tobacco like trichromes, roots, vascular tissues and pedicles. Therefore, these promoters may be used in cotton and other plants to derive gene expression in diverse tissues.

Conflict of Interest. The authors declare there no conflict of interest.

\section{References}

Aimin, W., Jinvuan, L. 2005. Analysis of the cotton E6 promoter. Tsinghua Science and Technology, 10: 409-413.

An, G., Watson, B.D., Chiang, C.C. 1986. Transformation of tobacco, tomato, potato and Arabidopsis thaliana using a binary Ti vector system. Plant Physiology, 81: 301-305.

Aso, T., Conaway, J.W., Conaway, R.C. 1994. Role of core promoter structure in assembly of the RNA polymerase-II preinitiation complex. A common pathway for formation of preinitiation intermediates at many TATA and TATA-less promoters. Journal of Biological Chemistry, 269: 26575-26583.

Benfey, P.N., Ren, L., Chua, N.H. 1990. Tissue-specific expression from CaMV $35 \mathrm{~S}$ enhancer sub-domains in early stages of plant development. $E M B O$ Journal, 9: 1677-1684.

Deikman, J. 1997. Molecular mechanisms of ethylene regulation of gene transcription. Physiologia Plantarum, 100: 561-566.

Deng, F., Tu, L., Tan, J., Li, Y., Nie, Y. Zhang, X. 2012. GbPDF1 is Involved in cotton fibre initiation via the core $c i s$-element HDZIP2ATATHB21. Plant Physiology, 158: 890-904.

Douliez, J., Michon, T., Marion, D. 2000. Steady-state tyrosine fluorescence to study the lipid-binding properties of a wheat nonspecific lipid-transfer protein (nsLTP1). Biochimica et Biophysica Acta, 1467: 65-72.

Ellis, J.G., Llewellyn, D.J., Walker, J.C., Dennis, E.S., Peacock, W.J. 1987. The ocs-element: a 16-base pair palindrome essential for activity of the 
octopinesynthase enhancer. EMBO Journal, 6: 3203-3208.

Giuliano, G., Pichersky, E., Malik, V.S., Timko, M.P., Scolnik, P.A., Cashmore, A.R. 1988. An evolutionary conserved protein binding sequence upstream of a plant light- regulated gene. Proceedings of the National Academy of Sciences of USA, 85: 7089-7093.

Gou, J.Y., Wang, L.J., Shuang-Ping, C., Wen-Li, H., Xiao-Ya, C. 2007. Gene expression and metabolite profiles of cotton fiber during cell elongation and secondary cell wall synthesis. Cell Research, 17: 422-434.

He, Z., Jiang, X., Qi, Y., Luo, D. 2008. Assessment of the utility of the tomato fruit- pecific E8 promoter for driving vaccine antigen expression. Genetica, 133: 207-214.

Hou, L., Liu, H., Li, J., Yang, X., Xiao, Y., Lou, M., Song, S., Yang, G., Pei, Y. 2008. SCFP, a novel fiber-specific promoter in cotton. Chinese Science Bulletin, 53: 2639-2645.

Hsu, C.Y., Creech, R.G., Jenkins, J.N., Ma, D.P. 1999. Analysis of promoter activity of cotton lipid transfer protein gene LTP6 in transgenic tobacco plants. Plant Science, 143: 63-70.

Iqbal, N., Asif, M., Masood, A., Naqvi1, A.Z., Khatoon, A., Bashir, A. 2017. Isolation and characterization of sucrose phosphate synthase promoter from cotton. Australian Journal of Crop Science AJCS, 11: 668-675.

John, M.E., Crow, L.J. 1992. Gene expression in cotton (Gossypium hirsutum L.) fiber: Cloning of the mRNAs. Proceedings of the National Academy of Sciences of USA, 89: 5769-5773.

John, M.E., Keller, G. 1996. Metabolic pathway engineering in cotton: biosynthesis of polyhydroxybutyrate in fibre cells. Proceedings of the National Academy of Sciences of USA, 93: 12768-12773.

Kim, K.Y., Kwon, S.Y., Lee, H.S., Hur, Y., Bang, J.W., Kwak, S.S. 2003. A novel oxidative stress-inducible peroxidase promoter from sweet potato: molecular cloning and characterization in transgenic tobacco plants and cultured cells. Plant Molecular Biology, 51: 831-838.

Kolchanov, N.A., Fgnatieva, E.V. Ananko, E., Podkolodyaya, O.A., Stepheneko, I.L. Merkulova, T.I., Pozdnyakov, M.A., Podkolodny, N.L., Naumochkin, A.N., Romashchenko, A.G. 2002. Transcription regulatory regions data base (TRRD): its status in 2002. Nucleic Acid Research,
30: 312-317.

Lescot, M., Dehais, P., Moreau, Y. 2002. PlantCARE: A database of plant cis-acting regulatory elements and a portal to tools for in silico analysis of promoter sequences. Nucleic Acids Research, 30: 325-327.

Li, X.B., Cai, L., Cheng, N.H., Liu, J.W. 2002. Molecular characterization of the cotton GhTUB1 gene that is preferentially expressed in fiber. Plant Physiology, 130: 666-674.

Li, X.B., Fan, X.P., Wang, X.L., Cai, L., Yang, W.C. 2005. The cotton $A C T I N 1$ gene is functionally expressed in fibres and participates in fibre elongation. Plant Cell, 17: 859-875.

Liu, H.C., Creech, R.G., Jenkins, J.N., Ma, D.P. 2000. Cloning and promoter analysis of the cotton lipid transfer protein gene Ltp3. Biochimica et Biophysica Acta, 1487: 106-111.

Masood, A, Iqbal, N., Mubeen, H., Naqvi, R.Z., Bashir, A. 2017. Cloning and expression analysis of Dhordein hybrid promoter isolated from barley (Hordeum vulgare L.). Pakistan Journal of Botany, 49: 1085-1095.

Mcknight, S., Tijian, R. 1986. Transcriptional selectivity of viral genes in mammalian cells. Cell, 46: $795-$ 805.

Paquis, S., Mazeyrat, F., Fernandez, O., Crouzet, J., Clement, C., Baillieul, F., Dorey, S. 2010. Characterization of a F-box gene up-regulated by phytohormones and upon biotic and abiotic stresses in grapevine. Molecular Biology Reports, 38: 3327 37.

Pu, L., Qun, L., Fan, X., Yang, W., Xue, Y. 2008. The R2R3 MYB transcription factor GhMYB109 is required for cotton fibre development. Genetics, 180: $811-820$.

Qin, L.X., Rao, Y., Li, L., Huang, J.F., Xu, W.L. 2013. Cotton GalT1 encoding a putative glycosyl transferase is involved in regulation of cell wall pectin biosynthesis during plant development. PLOS ONE, 8: e59115.

Rinehart, J.A., Peterson, M.W., John, M.E. 1996. Tissuespecific and developmental regulation of cotton gene FbL2A: demonstration of promoter activity in transgenic plants. Plant Physiology, 112: 13311341.

Salinas, J., Oeda, K., Chua, N.H. 1992. Two G-boxrelated sequences confer different expression patterns in transgenic tobacco. Plant Cell, 4: 1485 1493. 
Twell, D., Yamaguchi, J., McCormick, S. 1990. Pollenspecific gene expression in transgenic plants: coordinate regulation of two different tomato gene promoters during microsporogenesis. Development, 109: $705-713$.

Wingender, E., Chen, X., Fricke, E., Geffer, R., Hehl, R., Liebich, I., Krull, M., Matys, V., Michael, H., Ohnhauser, R., Pruss, M., Schacherer, F., Thiele, S., Urbach, S. 2001. The TRANSFAC system on gene expression regulation. Nucleic Acid Research, 29: 281-283.

Wu, A.M., Ling, C., Liu, J.Y. 2006. Isolation of a cotton reversibly glycosylated polypeptide (GhRGP1) promoter and its expression activity in transgenic tobacco. Journal of Plant Physiology, 163: 426435.

Wu, A.M., Lu, S.Y., Liu, J.Y. 2007. Functional analysis of a cotton glucuronosyl- transferase promoter in transgenic tobaccos. Cell Research, 17: 174183.

Wu, Y.F., Xu, W.L., Huang, G.Q., Gong, S.Y., Li, J. 2009. Expression and localization of GhH6L, a putative classical arabinogalactan protein in cotton (Gossypium hirsutum). Acta Biochimica Biophysica Sinica, 41: 495-503.

Yang, N.S., Russell, D. 1990. Maize sucrose synthase1 promotor directs phloem cell-specific expression of Gus gene in transgenic tobacco plants. Proceedings of the National Academy of Sciences of USA, 87: 4144-4148.

Yang, Y., Bian, S., Yao, Y., Liu, J. 2008. Comparative proteomic analysis provides new insights into the fiber elongating process in cotton. Journal of Proteome Research, 7: 4623-4637. 